\title{
Multilayer Laue Lens: A Path Toward One Nanometer X-Ray Focusing
}

\author{
Hanfei Yan, ${ }^{1,2}$ Hyon Chol Kang, ${ }^{3,4}$ Ray Conley, ${ }^{2,5}$ Chian Liu, ${ }^{5}$ Albert T. Macrander, ${ }^{5}$ \\ G. Brian Stephenson, ${ }^{1,3}$ and Jörg Maser ${ }^{1,4}$ \\ ${ }^{1}$ Center for Nanoscale Materials, Argonne National Laboratory, Argonne, IL 60439, USA \\ ${ }^{2}$ National Synchrotron Light Source II, Brookhaven National Laboratory, Upton, NY 11973, USA \\ ${ }^{3}$ Materials Science Division, Argonne National Laboratory, Argonne, IL 60439, USA \\ ${ }^{4}$ Department of Advanced Materials Engineering and BK21 Education Center of Mould Technology for Advanced Materials and Parts, \\ Chosun University, Gwangju 501-759, Republic of Korea \\ ${ }^{5}$ Advanced Photon Source, Argonne National Laboratory, Argonne, IL 60439, USA
}

Correspondence should be addressed to Hanfei Yan, hyan@bnl.gov

Received 12 May 2010; Accepted 13 September 2010

Academic Editor: Gene Ice

Copyright ( $) 2010$ Hanfei Yan et al. This is an open access article distributed under the Creative Commons Attribution License, which permits unrestricted use, distribution, and reproduction in any medium, provided the original work is properly cited.

\begin{abstract}
The multilayer Laue lens (MLL) is a novel diffractive optic for hard X-ray nanofocusing, which is fabricated by thin film deposition techniques and takes advantage of the dynamical diffraction effect to achieve a high numerical aperture and efficiency. It overcomes two difficulties encountered in diffractive optics fabrication for focusing hard X-rays: (1) small outmost zone width and (2) high aspect ratio. Here, we will give a review on types, modeling approaches, properties, fabrication, and characterization methods of MLL optics. We show that a full-wave dynamical diffraction theory has been developed to describe the dynamical diffraction property of the MLL and has been employed to design the optimal shapes for nanofocusing. We also show a $16 \mathrm{~nm}$ line focus obtained by a partial MLL and several characterization methods. Experimental results show a good agreement with the theoretical calculations. With the continuing development of MLL optics, we believe that an MLL-based hard x-ray microscope with true nanometer resolution is on the horizon.
\end{abstract}

\section{Introduction}

X-ray techniques have found numerous applications in life science, materials science, chemistry, medicine, and environmental science utilizing the unique properties of $\mathrm{X}$-rays, such as penetration capability and sensitivity to structural and chemical information. The rapid growth of nanoscience in the last decade gives rise to a strong demand for X-ray microscopy tools capable of providing information at the nanoscale. Many of the grand challenges we are facing today may be tackled only when such tools become available. For example, chemical imaging of spatial heterogeneities at nanoscale in real catalysts is essential to understand the diffusion of reactants and reaction products within the porous catalyst crystals or grains of submicrometer dimensions [1]. However, because of the weak refractive interaction of materials for X-rays (the difference of the refractive index from unity is typically $10^{-5}-10^{-6}$ for hard X-rays), it is very difficult to fabricate $\mathrm{X}$-ray nanofocusing optics. This difficulty is the major obstacle preventing current X-ray microscopy from achieving nanometer resolution.

There have been significant efforts devoted to the development of X-ray focusing optics utilizing the refraction, reflection and diffraction properties of X-rays. Recent progress in mirrors [2], zone plates (ZPs) [3], and refractive lenses [4] has pushed the frontiers of X-ray nanofocusing well below $50 \mathrm{~nm}$. However, many of these optics may be close to their practical limits for focusing. The fabrication of zone plates is limited by lithographic methods; it is hard to fabricate zone plate with below $15 \mathrm{~nm}$ outmost zone width, a requirement for a small focus, and with an adequately high aspect ratio required for high efficiency at hard X-ray energies. Refractive lens have achieved $\sim 50 \mathrm{~nm}$ [2] in two dimension (2D) [4], but a further reduction 


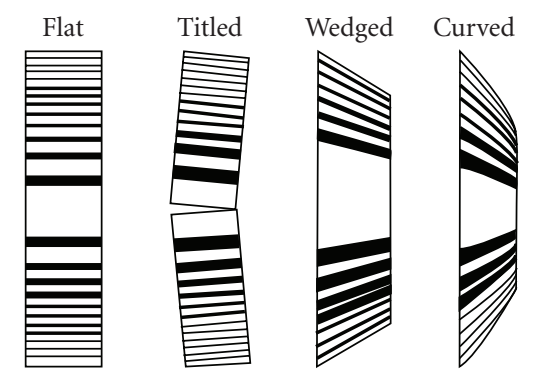

FIgURE 1: Types of multilayer Laue lens arrangements: flat, tilted, wedged, and curved. Reprinted from [18]. Copyright 2007, with permission from the American Physical Society.

of the focal size is very challenging, again because of the fabrication difficulty. Kirkpatrick-Baez (K-B) mirrors have made tremendous progress. Very recently, a line focus of $7 \mathrm{~nm}$ in one dimension (1D) has been achieved by multilayer K-B mirrors [5].

The multilayer Laue lens (MLL) [6, 7], a novel diffractive optic, has been shown in theory to be able to focus Xrays to well below $1 \mathrm{~nm}$ with very high efficiency. MLL is a special type of linear ZP which consists of thousands of alternating layers grown by the magnetron sputter deposition technique [8]. The thin film deposition method allows the growth of very thin zones with an almost limitless aspect ratio, overcoming the two difficulties encountered in the fabrication of lithographically produced ZP. MLL is a $1 D$ focusing optic. To date a line focus of $16 \mathrm{~nm}$ with a focusing efficiency over $30 \%$ has been achieved by a partial MLL structure at an energy of $19.5 \mathrm{keV}$ [9]. However, for real applications, two MLLs focusing in orthogonal directions have to be assembled to produce a $2 \mathrm{D}$ focus[10], similar to $\mathrm{K}-\mathrm{B}$ mirrors. Because an MLL is operated in transmission geometry and the size of the lens is on the order of tens of microns, assembling two MLLs to produce a $2 \mathrm{D}$ focus is feasible.

\section{Types of MLLs}

MLLs can be treated as special 1D zone plates with structures optimized for dynamical diffraction. They may be divided into four different types: the flat, tilted, wedged, and curved MLLs as shown in Figure 1. The MLL possessing flat zones is no different from a 1D ZP. It consists of thousands of alternating layers (zones) with alternating optical constants. The position of the $j$ th zone follows the zone plate law

$$
x_{j}^{2}=j \lambda f+\frac{j^{2} \lambda^{2}}{4},
$$

where $\lambda$ is the wavelength of the incident X-ray and $f$ is the focal length. According to the Rayleigh criterion, the diffraction-limited focus of a $1 \mathrm{D}$ lens is determined by

$$
s=\frac{0.5 \lambda}{N A}
$$

Here, $s$ is the focus size and $N A$ is the numerical aperture of the lens, approximately equal to $x_{\max } / f$. If one utilizes the full-width-at-half-maximum as the focus size, a smaller factor 0.44 in (2) should be used. By combining (1) with (2), one obtains

$$
s=\Delta x_{\min }, \quad \Delta x_{\min }=\frac{\lambda f}{2 x_{\max }} \sqrt{1+\frac{x_{\max }^{2}}{f^{2}}},
$$

where $\Delta x_{\min }$ is the outmost zone width of the lens. Thus, one has to fabricate a thinner outmost zone in order to achieve a smaller focus.

Conventionally, the focusing performance of a $\mathrm{ZP}$ is calculated by the geometric-optical theory, which neglects the X-ray diffraction effect inside the optic. The focusing efficiency for the $h$ th focusing order is given by [11]

$$
c_{h}=\left\{\begin{array}{r}
\frac{2 \exp (-2 k w \bar{\eta})}{h^{2} \pi^{2}}[\cosh (k w \Delta \eta)-\cos (k w \Delta \delta)], \\
h= \pm 1, \pm 3, \pm 5, \ldots, \\
h= \pm 2, \pm 4, \pm 6, \ldots,
\end{array}\right.
$$

where $k=2 \pi / \lambda, \Delta \delta=\delta_{A}-\delta_{B}, \Delta \eta=\eta_{A}-\eta_{B}$ and $\bar{\eta}=\left(\eta_{A}+\eta_{B}\right) / 2$. Here, $n_{A}=1-\delta_{A}+i \eta_{A}$ and $n_{B}=$ $1-\delta_{B}+i \eta_{B}$ are the refractive indexes of the lens materials $A$ and $B$ and $w$ is the section depth of the lens along its optical axis. For high efficiency, the phase change, $k w \Delta \delta$, associated with the optical path difference in zones made of materials $A$ and $B$ has to be close to (or equal to if there is no absorption) $\pi$ for maximum efficiency. However, this simple calculation is valid only when the lens is "optically thin"; that is, the section depth $w$ is less than $\left(2 \Delta x_{\min }\right)^{2} / \lambda$. At the optimum section depth, this condition is usually satisfied for zones with widths no smaller than $10 \mathrm{~nm}$. For thinner zones, the dynamical diffraction effect becomes dominant and the zones diffract X-rays more like crystals. Studies on a thick-sectioned multilayer have shown clearly the dynamical diffraction effect [12]. Therefore, an MLL that can focus $\mathrm{X}$-rays to below $10 \mathrm{~nm}$ and with reasonable efficiency is operating in the dynamical diffraction regime, akin to Laue diffraction in the transmission geometry. This property is very different from a conventional ZP. In such cases, the MLL performance is dependent on the titling angle because of the diffraction effect; an improvement is expected when the MLL is tilted by a small angle to satisfy the Bragg condition at one particular region within the MLL stack. This consideration leads to the tilted MLL.

For a tilted MLL, the Bragg condition is only fulfilled at a specific location of the lens because the zone width varies gradually from the center to the outmost region of the lens, limiting the maximum improvement that can be obtained by tilting alone. A further improvement can be achieved by a wedged MLL, in which zones are tilted progressively to the incident radiation. The approximate Bragg condition is satisfied everywhere if the numerical aperture (NA) of the lens is moderate. To achieve a focus size close to the wave length, a parabolic (plane wave illumination) or elliptical (spherical wave illumination) zone profile is required to exactly fulfill the Bragg condition. We will have a detailed discussion on the properties of different types of MLLs in the following sections. 


\section{Simulation Methods}

As has been discussed in the preceding section, the geometrical theory becomes invalid for an MLL with a high NA. For this type of volume diffractive optic, the transmission function at the exit surface of the lens cannot be obtained by a simple ray tracing calculation of the flight path of the incident X-rays. One has to employ a full-wave diffraction theory to study the propagation and diffraction of X-ray waves inside the lens. This was initially done by Maser and Schmahl [13], who applied locally a 1D approach of the coupled-wave theory (CWT) to study the variation of the local diffraction with respect to the thickness and slant of the zones. With the assumption that the MLL can be locally decomposed into periodic gratings, their approach is limited to cases of $w \ll f$ with a relatively small NA corresponding to a resolution of $2 \mathrm{~nm}$. Later on and using similar assumptions, Levashov and Vinogradov studied the variation of the total diffraction efficiency with thickness [14]. A numerical approach based on a parabolic wave equation with the paraxial approximation was developed by Kurokhtin and Popov [15]. More recent methods of solving the parabolic wave equation using eigenfunctions have been reported $[16,17]$. The validity of these approaches is limited to a relatively small NA due to the paraxial approximation. To overcome the limitation of these approaches and to provide a model that is valid to a spatial resolution on the order of the wavelength of radiation used, we developed a modeling method that is analogous to Takagi-Taupin equations in crystallography by realizing the similarities of X-ray diffraction between an MLL and a single crystal [18].

The scalar wave equation describing the electric field variation of a monochromatic $\mathrm{X}$-ray wave in a medium with susceptibility function $\chi(\mathbf{r})$ is given by

$$
\nabla^{2} \mathbf{E}(\mathbf{r})+k^{2}[1+\chi(\mathbf{r})] \mathbf{E}(\mathbf{r})=0 .
$$

We begin by considering an MLL with flat zones whose positions satisfy (1). After a variable transform,

$$
x^{\prime}=\sqrt{x^{2}+f^{2}}-f,
$$

the structure of the MLL becomes periodic in terms of $x^{\prime}$ so that the susceptibility function can be expanded into a puesdo-Fourier series in terms of $x$

$$
\begin{aligned}
& \chi(x)=\sum_{h=-\infty}^{\infty} \chi_{h} \exp \left[i \phi_{h}(x)\right], \quad \chi_{0}=\frac{\chi_{A}+\chi_{B}}{2}, \\
& \chi_{h \neq 0}=\frac{\chi_{A}-\chi_{B}}{2 i h \pi}\left[1-(-1)^{|h|}\right], \quad \phi_{h}=h k\left(\sqrt{x^{2}+f^{2}}-f\right) .
\end{aligned}
$$

For an incident wave, $\mathbf{E}_{0}^{(a)}(\mathbf{r}) \exp \left(i \mathbf{k}_{0} \cdot \mathbf{r}\right)$, we assume that a trial solution to the wave equation can be written as

$$
\mathbf{E}(\mathbf{r})=\sum_{h=-\infty}^{\infty} \mathbf{E}_{h}(\mathbf{r}) \exp \left[i\left(\mathbf{k}_{\mathbf{0}} \cdot \mathbf{r}+\phi_{h}\right)\right] .
$$

Substituting (7) and (8) into (5) and equating terms with the same order $h$ yield an infinite set of differential equations.
We further simplify the system by neglecting second-order derivatives on $\mathbf{E}_{h}(\mathbf{r})$ and limiting the number of excited orders. The validity of these approximations is discussed in [18]. Eventually, we arrive at a set of differential equations

$$
\begin{gathered}
\frac{2 i}{k} \nabla E_{h} \cdot\left(\mathbf{s}_{0}+\frac{\nabla \phi_{h}}{k}\right)+\beta_{h}(\mathbf{r}) E_{h}+\sum_{l} \chi_{h-l} E_{l} \cos \vartheta_{h l}=0, \\
\beta_{h} \approx \frac{k^{2}-\left(k_{0}+\nabla \phi_{h}\right)^{2}}{k^{2}}, \quad h, l=0, \pm 1, \pm 2, \ldots,
\end{gathered}
$$

with the boundary conditions

$$
\left.\mathbf{E}_{0}(\mathbf{r})\right|_{\text {entrance }}=\left.\mathbf{E}_{0}^{(a)}(\mathbf{r})\right|_{\text {entrance }},\left.\mathbf{E}_{h \neq 0}(\mathbf{r})\right|_{\text {entrance }}=0 .
$$

Here, $\mathbf{s}_{0}$ is a unit vector along the incident direction and $\vartheta_{h l}$ is the angle between the polarization direction of the $h$ th and $l$ th diffraction orders. The quantity, $\beta_{h}$, represents the deviation from the Bragg condition for the $h$ th diffraction order. For other types of MLLs, (9) still holds but $\phi_{h}$ will possess a different functional form.

\section{Properties of MLL}

We have mentioned in the preceding section that the geometric theory is no longer valid for thick MLLs. In the following, we will employ the theoretical model in Section 3 to study the wave propagation and diffraction inside a thick MLL.

We start by considering a MLL with flat zones and a radius, $x_{\max }$, of $30 \mu \mathrm{m}$. At $19.5 \mathrm{keV}(=0.634 \lambda \AA)$, it has a focal length $\mathrm{f}$ of $4.72 \mathrm{~mm}$ and an outmost zone width of $5 \mathrm{~nm}$. The lens is made of $\mathrm{WSi}_{2}$ and $\mathrm{Si}$. An incident plane wave with $\sigma$ polarization impinges on the MLL with an angle $\theta$ to its surface normal, as shown in Figure 2. A $\theta$ angle of zero degree corresponds to the normal incidence. Because $x_{\max }$ is much smaller than $f$, one can neglect the second term on the right hand side of (1) and arrive at

$$
\phi_{h} \approx \frac{h \pi}{\lambda f} x^{2}, \quad \beta_{h} \approx-2 \frac{h x}{f} \sin \theta-\left(\frac{h x}{f}\right)^{2} .
$$

Substituting this into (9) and limiting the calculation to only consider a finite number of diffraction orders, we can numerically solve the exit wave front for different diffraction orders. In our definition, a diffraction order with a negative sign corresponds to a converging wave, so that the negative first order is the primary focusing order and is of most interest.

To begin, we will study the dependence of the local diffraction intensity at the exit surface of the lens, $\mid E_{-1}(x$, $w)\left.\right|^{2}$, on the section depth, $w$, under the condition of normal incidence $(\theta=0)$. Two values, $w=1.5$ and $w=10 \mu \mathrm{m}$, are considered. These two cases correspond to a thin and thick lens, respectively. In the latter case, the phase change in two adjacent zones is $\pi$. The geometrical theory is invalid for a thick lens, and the dynamical diffraction model has to be employed. In the top panel of Figure 3(a), we plot the variation of the local diffraction intensity of the negative 


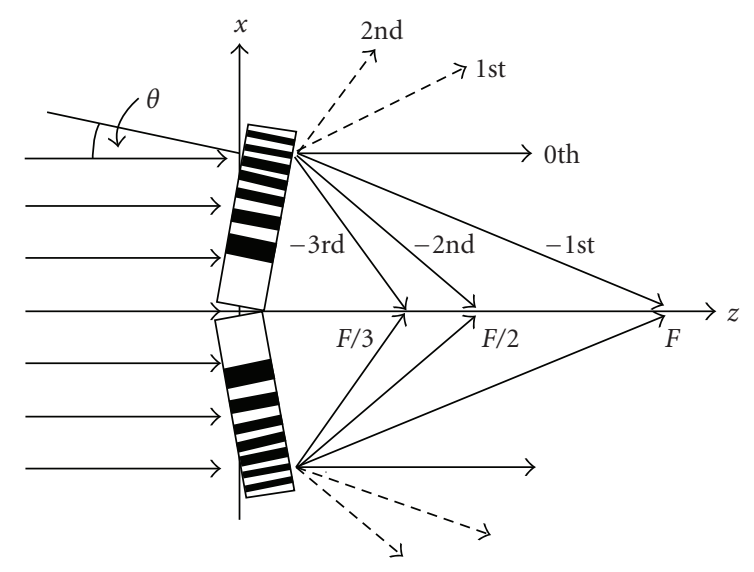

FIGURE 2: Schematic representation of a plane wave diffracted with a tilted MLL containing flat zones, in which many diffraction orders are excited. Reprinted from [18]. Copyright 2007, with permission from the American Physical Society.

first order calculated by the dynamical diffraction model (9) and the geometric model (4). One can clearly see that for the thin lens, both models yield almost identical results, but for the thick lens, they differ significantly in most regions except in the vicinity of the center where the thin lens approximation is still valid. For the thin lens, although the local diffraction intensity is low, it is nearly a constant across the lens. Therefore, all parts of the lens contribute equally to focusing and are effective. As a result, the thin lens' effective NA is equal to its physical NA, $x_{\max } / f$, as predicted by the geometric theory. In contrast, the local diffraction intensity of a thick lens drops down quickly to almost zero in the outer region. Lens positions beyond $x=15 \mu \mathrm{m}$ do not contribute to focusing as strongly as the inner parts. As a result, the effective NA of a thick MLL with flat zones is limited by the dynamical diffraction effect. A further increase in the physical NA of such a thick lens will not lead to a smaller focus in contrast to what happens for a thin lens.

Next, we will study the dependence of the lens' performance on the tilting angle. In the bottom panel of Figure 3(a), we plot the variation of the local diffraction intensity at a tilting angle $\theta$ of 1.6 milliradian. For the thick lens, a diffraction peak is observed around the position $x=$ $15 \mu \mathrm{m}$, where the Bragg condition is satisfied. The diffraction intensity drops down to zero around $x=25 \mu \mathrm{m}$, much larger than that under the condition of normal incidence. Due to this tilting, one can see that not only is the efficiency enhanced, but also the effective NA is increased. For the thin lens, tilting has very little effect on its performance, as expected. We also plot the focusing efficiency of the negative first order (the normalized integrated diffraction intensity) as a function of both the depth and the tilting angle (Figure 3(b)). It can be seen that at a section depth of $13.5 \mu \mathrm{m}$ and tilting angle of 2.1 milliradian a maximum focusing efficiency of $33 \%$ is reached. This efficiency is much higher than that in the conventional geometry (normal incidence, $\theta=0$ ), which is about $9.2 \%$. In addition, the focus size is reduced by tilting because the effective NA is increased.
Figure 4 depicts the focus profiles at four different tilting angles at the same section depth of $13.5 \mu \mathrm{m}$. This plot clearly shows the effect of tilting. At the angle of 2.1 milliradian, one achieves the smallest focus with the highest efficiency.

We have shown that for an MLL with flat zones, the effective NA may be limited, depending on whether the dynamical diffraction effect becomes dominant. For an MLL with a very small outmost zone width, one can always achieve a diffraction-limited focus size if the section depth of the lens is small enough so that the thin lens approximation is valid. However, the efficiency has to be sacrificed. There is a tradeoff between the effective NA and the efficiency for this type of MLL. For instance, for an MLL with $1 \mathrm{~nm}$ outmost zone width, the achievable focus size and the efficiency vary with the depth $w$ (Figure 5). In order to achieve a diffractionlimited focus size of $1 \mathrm{~nm}$, the lens has to be thinner than $0.5 \mu \mathrm{m}$ but its efficiency will decrease below $0.1 \%$. Because of the extremely low efficiency, such a lens may not be useful in practice.

The conflict between the effective NA and the efficiency can be overcome by optimizing the MLL structure for dynamical diffraction; that is, each zone is progressively tilted to satisfy the Bragg condition. This consideration results in a wedged zone structure as shown in Figure 1. The Bragg condition is approximately fulfilled everywhere in a wedged MLL satisfying the modified zone plate law

$$
x_{j}^{2}=\left(j \lambda f+\frac{j^{2} \lambda^{2}}{4}\right) a(z)^{2}, \quad a(z)=1-\frac{z}{2 f} .
$$

Accordingly, the phase function, $\phi_{h}$, is changed to

$$
\phi_{h}=h k\left[\sqrt{x^{2} / a(z)^{2}+f^{2}}-f\right] .
$$

For a wedged MLL with $1 \mathrm{~nm}$ outmost zone width and with a focal length of $2.6 \mathrm{~mm}$, we plot the local diffraction intensity of the zeroth, negative first, negative second, and negative third orders in Figure 6(a). The section depth is $16 \mu \mathrm{m}$, equal to the Pendellösung thickness for the maximum diffraction in the Laue geometry, $w=\lambda / 2 \sqrt{\left|\chi_{-1} \chi_{1}\right|}$. Within a close vicinity of the center, a low diffraction intensity of about 0.13 is observed and is in good agreement with the geometric calculation. This is because the lens in this region can still be considered thin. Around the position with a zone width of $20 \mathrm{~nm}(x=4 \mu \mathrm{m})$, the diffraction intensity jumps to about 0.7 and remains nearly a constant across the lens. The enhancement of the diffraction is due to the fulfillment of the Bragg condition so that strong dynamical diffraction is excited. Except in the region near the center, all other orders including the zeroth order (directly transmitted wave) are significantly suppressed. This is one of the great advantages of the wedged MLL because an order sorting aperture that blocks high orders may not be necessary. We obtain a diffraction-limited focus size (full-width-at-half-maximum) of $0.86 \mathrm{~nm}$ (Figure 6(b)), and a very high efficiency of 63\% for the negative first order, which is much higher than the maximum efficiency achievable even by a conventional phase ZP. 


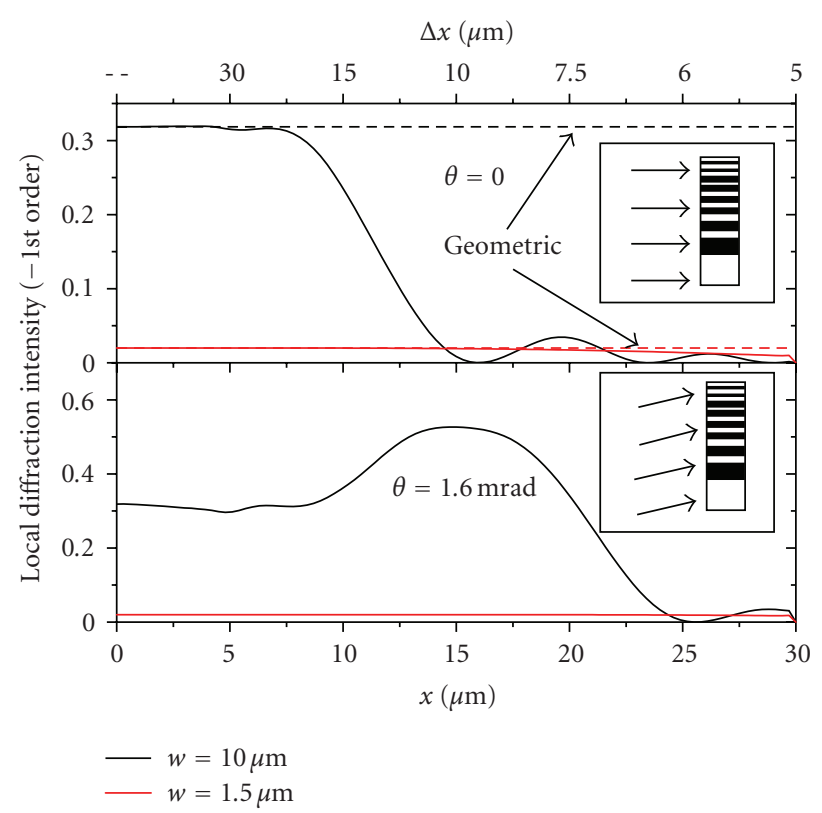

(a)

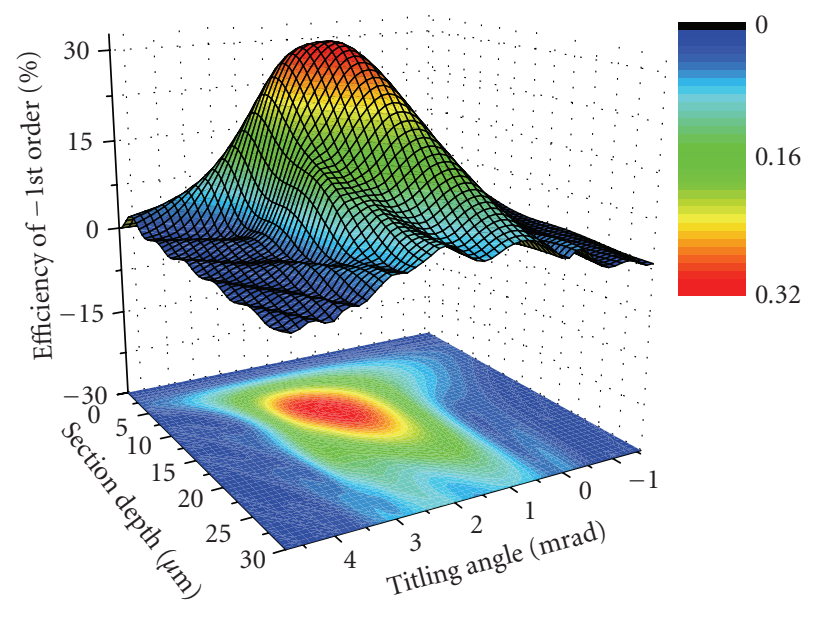

(b)

FIgURE 3: (a) The local diffraction intensity of the negative first order at the exit surface of the MLL with a section depth of 1.5 and $10 \mu \mathrm{m}$ in the normal incidence and tilted geometries. The dashed lines are calculated by the geometrical theory (4) in the cases of thick section (black) and thin section (red), assuming a normal incidence. (b) Efficiency of the negative first order as a function of the tilting angle and the section depth.

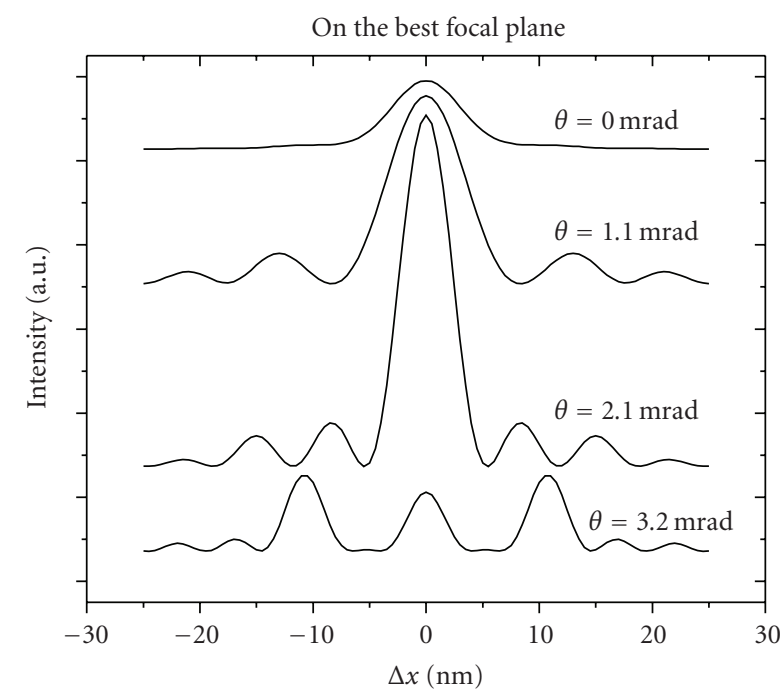

FIGURE 4: Focus profiles at four different tilting angles. Reprinted from [18]. Copyright 2007, with permission from the American Physical Society.

The wedged structure is still an approximation to the ideal MLL. In order to achieve a focus close to the wave length, elliptical (spherical wave illumination) or parabolic (plane wave illumination) zone profiles are needed. In such an MLL, the Bragg condition is satisfied exactly everywhere and the diffracted waves from each zone are in phase at the focus. Although the parabolic and elliptical profiles

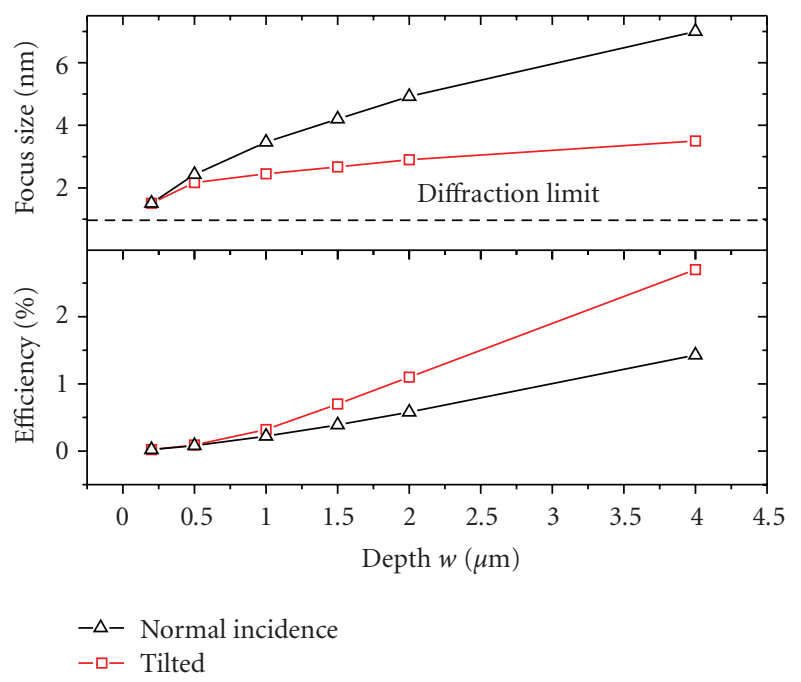

Figure 5: The efficiency and the focus size of an MLL with $1 \mathrm{~nm}$ outmost zone width at different section depths.

for multilayer focusing optics are well known and can be obtained from holography [19], the rigorous dynamical diffraction simulation shows that they are ideal as well for volume diffractive optics [18].

Real MLLs always possess imperfections. For example, a small growth error can result in a systematic deviation of the zone position from the zone plate law. This kind of error can be described by a zone plate equation with additional error terms [20] and can be incorporated into the simulation 


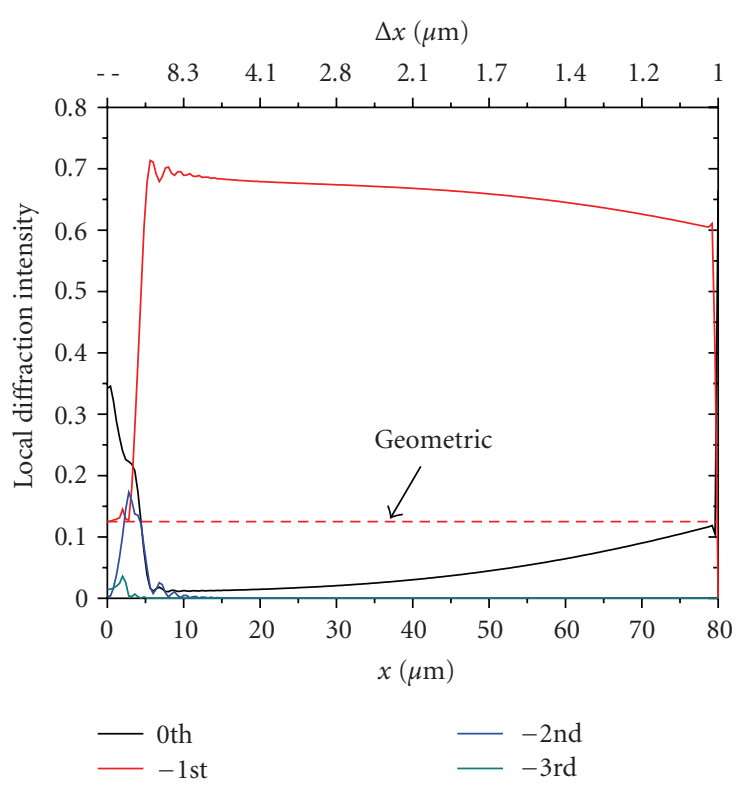

(a)

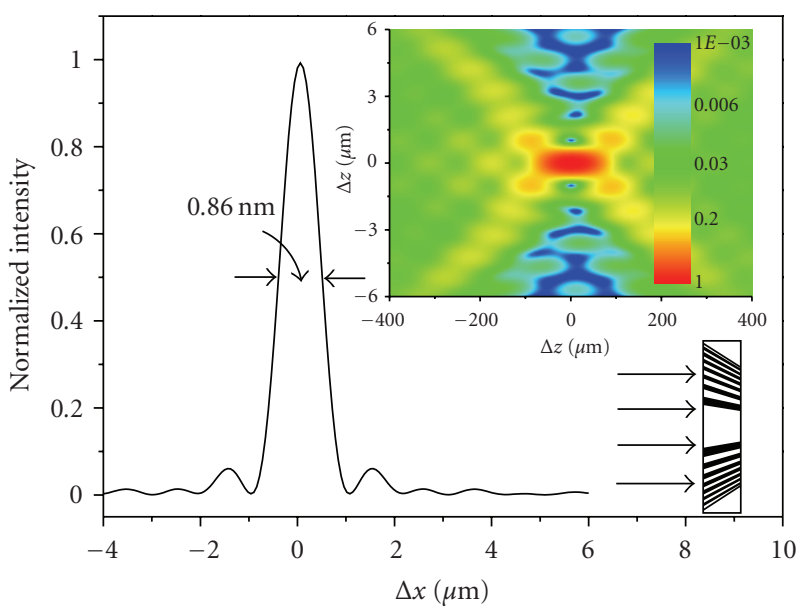

(b)

Figure 6: (a) The local diffraction intensity of the zeroth, negative first, second, and third orders of a wedged MLL with $1 \mathrm{~nm}$ outmost zone width. The dashed line is calculated by (4) and is for the negative first order. (b) The focus profile shows a diffraction-limited size of $0.86 \mathrm{~nm}$. The inset on top is the isophote pattern near the focus (in logarithmic scale).

model by changing the phase function, $\phi_{h}$, accordingly. With this type of imperfection, the focusing efficiency is usually nearly unchanged, but the focus is greatly distorted. Another type of imperfection is due to interfacial roughness, which is inevitable with layer thicknesses approaching atomic dimensions. If the roughness is uncorrelated, its effect is accounted for by a roughness factor similar to "DebyeWaller factor", $\exp \left(-M_{h}\right)=\left\langle\exp \left(i \boldsymbol{\rho}_{h} \cdot \mathbf{u}\right)\right\rangle$, where $\boldsymbol{\rho}_{h}=$ $\nabla \phi_{h}$ is the $h$ th local reciprocal-lattice vector, $\mathbf{u}$ is the random displacement vector of the interface, and the angle bracket denotes the statistical average [21]. The pseudoFourier coefficient of the susceptibility function, $\chi_{h}$, has to be multiplied by this factor. Uncorrelated roughness usually results in a decrease of the focusing efficiency but has little effect on the focus profile, unless the RMS roughness becomes comparable with the zone width.

\section{Fabrication Methods}

The MLL growth was performed using DC magnetron sputtering systems located in the deposition laboratories of both the Advanced Photon Source (APS) [22] and the National Synchrotron Light Source-II (NSLS-II). To ensure the accuracy of the layer position, the thinnest layer is deposited first, which lessens the impact of any buildup in the thickness error during the growth. The multilayer structures were deposited on both diced Si (100) semiconductorgrade substrates and superpolished Si (100) substrates. The selection of substrate doping concentration was not closely controlled beyond a preference for low resistivity in order to assist with electron dissipation during postsectioning SEM analysis.
MLL deposition employed standard 3 inch MAK and Onyx-3 cathodes at the APS, and modified Polaris [23] 3 inch direct-gas injection cathodes at the NSLS-II. We chose the $\mathrm{Si} / \mathrm{WSi}_{2}$ materials system for several reasons. We have found that there is a compensation of compressive stresses in Si layers by tensile stresses in $\mathrm{WSi}_{2}$ layers, with very little net accumulation [24]. The low buildup stress avoids the occurrence of delamination. In-situ X-ray reflectivity study also revealed that the noticeably rough $\mathrm{Si}$ layer can be sharpened by the $\mathrm{WSi}_{2}$ layer [25], leading to interfaces with very small roughness. The speed of sputtering is another concern. The Si film growth rate is $10 \AA$ to $13 \AA \cdot \mathrm{sec}^{-1}$, and the WSi $i_{2}$ film growth rate is $30 \AA$ to $40 \AA \cdot \mathrm{sec}^{-1}$, which are reasonably fast. Other than this system, other systems are being explored actively elsewhere for the MLL growth $[26,27]$.

For our MLL deposition, the $\mathrm{WSi}_{2}$ targets used are 99.5\% pure powder-hot-pressed with a nominal bulk density of $8.10 \mathrm{~g} \cdot \mathrm{cm}^{-3}$, and the Si targets used are $99.999 \%$ pure and Boron-doped to allow for DC sputtering. No electrical bias or active temperature control is presently applied to the substrates; however, the substrate temperature does rise somewhat above ambient due to the presence of the plasma and energetic ion bombardment. The target to sample distance used varied from $70 \mathrm{~mm}$ to $110 \mathrm{~mm}$. A base pressure of $10^{-8}$ Torr is reached before the commencement of pump throttling and Ar process gas injection. Ar gas pressure is held at a constant 2.3 milliTorr (with later growths using 4 milliTorr) by upstream MFC feedback control. Deposition was carried out using a constant cathode power of 215 watts for both guns, and each gun is turned on for 7 seconds before the start of each layer growth to stabilize. Uniform deposition 
thickness is affected by raster-scanning the substrate over figured apertures by varying both the number of passes over this aperture and the translational velocity. Custom software controls design each pass over the sputtering gun to deposit as close as possible to $5 \AA$ of film growth, or $10 \AA$ per loop. The individual layer thickness requirement is then divided by the calculated number of loops, and the translational velocity is adjusted as needed. As the required thickness changes, additional passes over the appropriate sputtering gun and translational velocity adjustments are handled automatically.

Wedged MLL growth uses the same deposition process as described above but with sharply tapered masks to produce steep laterally graded layers [28]. Due to the inherent nature of magnetron deposition growth rate to decay over time, a compensation factor is included during the growth which adjusts the velocity appropriately. An initial MLL growth is characterized by extracting an inverse d-spacing line profile from SEM images [29] which is then used as feedback for a revised compensation factor for a second, accurate MLL growth.

Subsequent to growth, the multilayer must be sectioned and thinned to the requisite optical depth to form the halves of the MLL. A series of steps akin to that for making cross-section transmission electron microscopy samples has been found to be successful in making sections of sufficient perfection [30].

The physical aperture of the MLL is determined by the total deposition thickness. Currently, it is in the range of $10-40 \mu \mathrm{m}$ and is mainly limited by two factors: (1) the accumulated interfacial stress as the deposition thickness increases and (2) the maximum deposition allowed by the consumable sputtering target. The former can be controlled by properly setting the growth parameters so that the stresses generated in two adjacent layers have opposite sign and cancel each other. The latter can be addressed by adding more sputtering targets. A new sputtering deposition machine with eight guns has been built at NSLS-II to grow MLLs with sizes over $100 \mu \mathrm{m}$ [31], which will be comparable to the diameter of the high-resolution $\mathrm{ZP}$ that is currently available. This size is also in the same order of the coherence length of the thirdgeneration synchrotron source so that the lens will be able to fully utilize the coherent flux delivered to it.

\section{Characterization Methods}

A typical setup of the line focus measurement for MLLs is shown in Figure 7. A beam-defining aperture is used to reduce the size of the incident plane wave to match the size of the lens. A fluorescence detector is used to record the excited fluorescence signal from the scanning object, and a scintillation detector with narrow slits in front is placed at a downstream position to record the transmission and diffraction signals. The lens tested has a focal length of $2.6 \mathrm{~mm}$ at $19.5 \mathrm{keV}$, an outmost zone width of $5 \mathrm{~nm}$ and a deposited thickness of $13.5 \mu \mathrm{m}$, corresponding to about $40 \%$ of the full structure. Figure 8 is a scanning electron microscopy image of the lens, showing 1588 zones with zone widths varying from 25 to $5 \mathrm{~nm}$. Because the line focus is formed at the real optical axis through the center of the lens

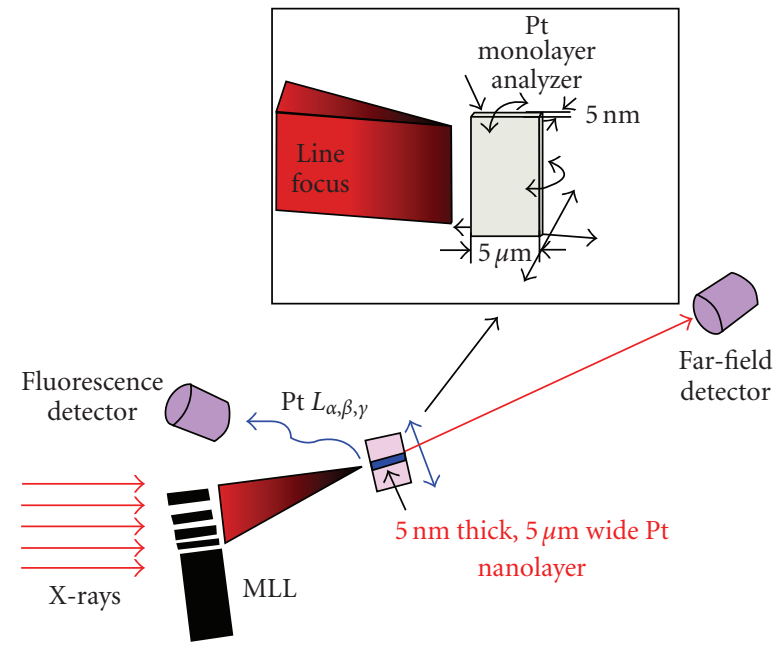

FIGURE 7: The experimental setup for the line focus measurement. The nanolayer has to be aligned parallel to the line focus in $\mathrm{x}$ and $\mathrm{z}$ directions.

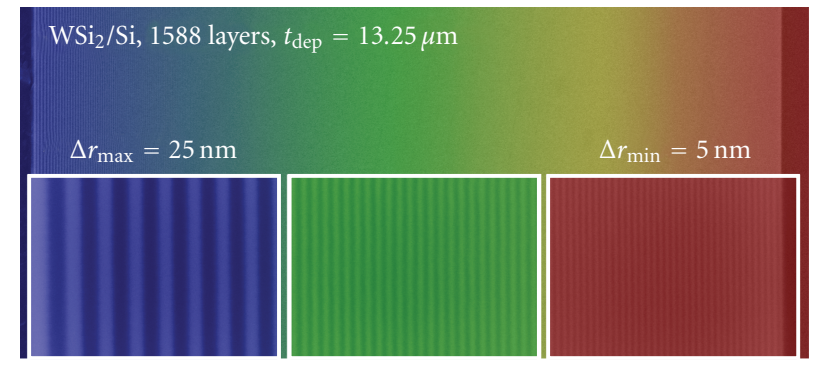

FIGURE 8: A scanning electron microscopy image of the MLL under test. The color is for aid to view and has no physical meanings. The white boxes on the bottom show the zoom-in images of the multilayer at three different locations. Reprinted from [9]. Copyright 2008, with permission from the American Institute of Physics.

and is several microns away from the directly transmitted wave, a beam-stop and an order sorting aperture are not required if the scanning object is smaller than this separation distance.

We first need to tilt the lens to the right angle for an optimum performance. This can be done by monitoring the variation of the transmission intensity as a function of the tilting angle. The top panel of Figure 9 depicts the transmission rocking curve, which has two symmetric attenuation dips around $\pm 0.1^{\circ}$. These two dips are ascribed to both the photoelectric absorption and the diffraction extinction effect. A theoretical simulation is also presented. One needs to make sure that the lens is tilted to the right direction so that the diffraction order for focusing, not for diverging, is enhanced. When the tilting angle becomes too large, significantly dissimilar from the Bragg condition for any d-spacing of the lens, the diffraction extinction becomes negligible. The difference of the attenuation at and off the Bragg condition is approximately equal to the focusing efficiency of the negative first order, since most of 


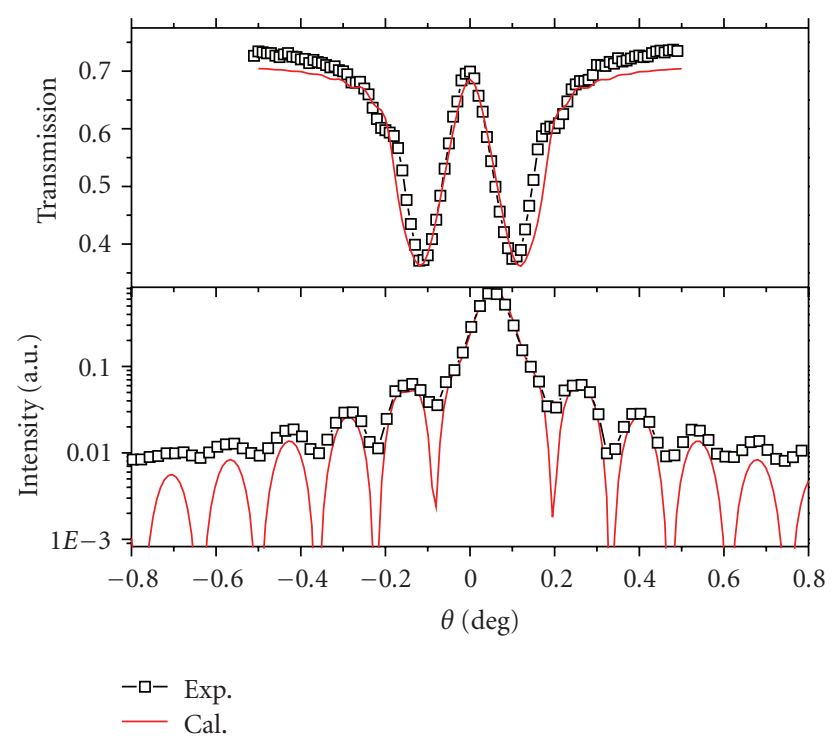

Figure 9: The transmission (top) and the diffraction (bottom) rocking curves.

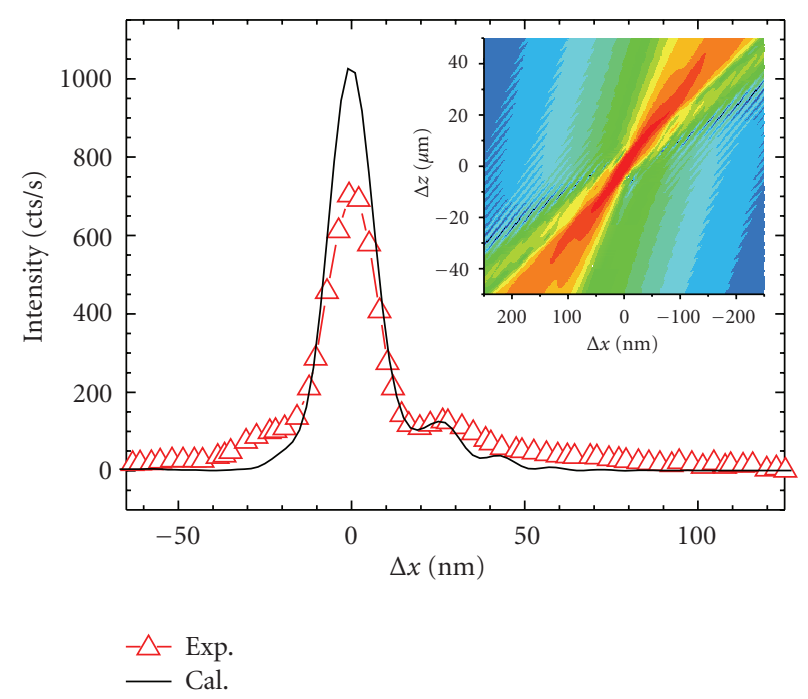

Figure 10: The measured line focus profile compared to the theoretical calculation. The inset shows the simulated isophote pattern near the focus. Reprinted from [9]. Copyright 2008, with permission from the American Institute of Physics.

the diffracted photons are focused to this order. From the measurement, an efficiency of $37 \%$ is observed.

We then move the scintillation detector along the $\mathrm{x}$-axis to a position a few millimeters away from the optical axis to measure the variation of the diffraction intensity at a specific $2 \theta$ angle as the lens is rocked. The bottom panel of Figure 9 shows one of these diffraction rocking curves, together with the theoretical simulation. At a fixed $2 \theta$ angle, rocking the lens results in a change of the scattering vector, $\Delta \mathbf{Q}$, perpendicular to the reciprocal lattice vector. Because the lens diffracts X-rays in the transmission geometry (Laue case), its reciprocal lattice vector is perpendicular to its depth direction (z-axis). As a result, the scanned $\mathbf{Q}$ direction $(\Delta \mathbf{Q})$ is along the $\mathrm{z}$-axis and the period of the intensity oscillation is determined by the section depth. Such a phenomenon is known as thickness fringes in crystal diffraction [32]. From this curve, we obtain a section depth of $13.4 \mu \mathrm{m}$.

A Platinum nanolayer with $5 \mathrm{~nm}$ thickness and $5 \mu \mathrm{m}$ depth along the optical axis is used as the scanning object to measure the line focus profile. This depth is chosen to maximize the fluorescence signal; however, it also has to be smaller than the depth of focus, which is $9.4 \mu \mathrm{m}$ in this case. The nanolayer needs to be well aligned with respect to the line focus so as to obtain the correct focus profile. In addition to the requirement of having a spatial placement exactly at the focal plane, the nanolayer also has to be aligned parallel to the line focus in both $\mathrm{x}$ and $\mathrm{z}$ directions (see Figure 7). The experimental result is shown in Figure 10, and is compared to the theoretical calculation. Although the diffraction-limited focus size is $12.5 \mathrm{~nm}$ for this lens, a $15 \mathrm{~nm}$ focus is expected from the simulation due to the dynamical diffraction effect at this section depth, which limits the effective NA. The measured focus is $16 \mathrm{~nm}$, very close to the theoretical prediction.

\section{Conclusion}

Hard X-ray imaging with nanometer resolution has been identified as one of the key objectives for making full use of the continuing investment in major synchrotron facilities [33]. The key to realizing this challenging goal is the successful development of nanofocusing optics for hard Xrays. We provide a review on types, modeling approaches, properties, and fabrication and characterization methods of MLL optics and show that MLLs are well suited for hard Xray nanofocusing with high efficiency. Sputtering deposition has demonstrated the capability of growing multilayers with layer thicknesses below $1 \mathrm{~nm}$ and with small interface roughness, making the fabrication of $1 \mathrm{~nm}$ MLL optics possible [22].

To date, a line focus of $16 \mathrm{~nm}$ with very high focusing efficiency has been obtained by MLL optics. For most real applications, however, a $2 \mathrm{D}$ focus is required. This can be achieved by placing two MLLs orthogonally [10], but the performance of the crossed pair is sensitive to many misalignments [34]. There has been great progress in the effort of 2D focusing by two crossed MLLs as well. An apparatus with eight degrees of freedom required for a full alignment of two MLLs has been designed and constructed $[35,36]$. A $2 \mathrm{D}$ imaging resolution of sub- $30 \mathrm{~nm}$ has been demonstrated using the MLL microscope [37]. With the continued development of MLL optics, we believe that hard $\mathrm{X}$-ray microscopy with true nanometer resolution is on the horizon.

\section{Acknowledgments}

Work at Argonne, including use of the Advanced Photon Source and Center for Nanoscale Materials, was supported by the Department of Energy, Office of Basic Energy Sciences under Contract no. DE-AC-02-06CH11357. 
Work at Brookhaven was supported by the Department of Energy, Office of Basic Energy Sciences under Contract no. DE-AC-02-98CH10886. One of the authors (H. C. Kang) would like to acknowledge the support by Basic Science Research Program through the National Research Foundation of Korea (NRF) funded by the Ministry of Education, Science, and Technology (MEST no. R15-2008006-01000-0 and no. 2010-0023604).

\section{References}

[1] B. M. Weckhuysen, "Chemical imaging of spatial heterogeneities in catalytic solids at different length and time scales," Angewandte Chemie - International Edition, vol. 48, no. 27, pp. 4910-4943, 2009.

[2] H. Mimura, H. Yumoto, S. Matsuyama et al., "Efficient focusing of hard x rays to $25 \mathrm{~nm}$ by a total reflection mirror," Applied Physics Letters, vol. 90, no. 5, Article ID 051903, 3 pages, 2007.

[3] W. Chao, B. D. Harteneck, J. A. Liddle, E. H. Anderson, and D. T. Attwood, "Soft X-Ray microscopy at a spatial resolution better than 15 nm," Nature, vol. 435, no. 7046, pp. 1210-1213, 2005.

[4] C. G. Schroer, O. Kurapova, J. Patommel et al., "Hard X-Ray nanoprobe based on refractive X-Ray lenses," Applied Physics Letters, vol. 87, no. 12, Article ID 124103, 3 pages, 2005.

[5] H. Mimura, S. Handa, T. Kimura et al., "Breaking the $10 \mathrm{~nm}$ barrier in hard-X-Ray focusing," Nature Physics, vol. 6, no. 2, pp. 122-125, 2010.

[6] H. C. Kang, J. Maser, G. B. Stephenson et al., "Nanometer linear focusing of hard X Rays by a multilayer laue lens," Physical Review Letters, vol. 96, no. 12, Article ID 127401, 4 pages, 2006.

[7] A. T. Macrander, H. Yan, and H. C. Kang, "Nanofocusing of hard X-Rays with multilayer Laue lenses," in Hand Book of Optics, vol. 5, McGraw-Hill, New York, NY, USA, 2009.

[8] C. Liu, R. Conley, A. T. MacRander et al., "Depth-graded multilayers for application in transmission geometry as linear zone plates," Journal of Applied Physics, vol. 98, no. 11, Article ID 113519, 6 pages, 2005.

[9] H. C. Kang, H. Yan, R. P. Winarski et al., "Focusing of hard X-Rays to 16 nanometers with a multilayer Laue lens," Applied Physics Letters, vol. 92, no. 22, Article ID 221114, 3 pages, 2008.

[10] J. Maser, G. B. Stephenson, S. Vogt et al., "Multilayer Laue lenses as high-resolution X-Ray optics," in Design and Microfabrication of Novel X-Ray Optics II, vol. 5539 of Proceedings of SPIE, Denver, Colo, USA, August 2004.

[11] J. Kirz, "Phase zone plates for X Rays and the extreme uv," Journal of the Optical Society of America, vol. 64, no. 3, pp. 301309, 1974.

[12] H. C. Kang, G. B. Stephenson, C. Liu et al., "High-efficiency diffractive X-Ray optics from sectioned multilayers," Applied Physics Letters, vol. 86, no. 15, Article ID 151109, 3 pages, 2005.

[13] J. Maser and G. Schmahl, "Coupled wave description of the diffraction by zone plates with high aspect ratios," Optics Communications, vol. 89, no. 2-4, pp. 355-362, 1992.

[14] V. E. Levashov and A. V. Vinogradov, "Analytical theory of zone plate efficiency," Physical Review E, vol. 49, no. 6, pp. 5797-5803, 1994.

[15] A. N. Kurokhtin and A. V. Popov, "Simulation of highresolution X-Ray zone plates," Journal of the Optical Society of America A, vol. 19, no. 2, pp. 315-324, 2002.
[16] C. G. Schroer, "Focusing hard X Rays to nanometer dimensions using Fresnel zone plates," Physical Review B, vol. 74, no. 3, Article ID , 4 pages, 2006.

[17] F. Pfeiffer, C. David, J. F. van der Veen, and C. Bergemann, "Nanometer focusing properties of Fresnel zone plates described by dynamical diffraction theory," Physical Review B, vol. 73, no. 24, Article ID 245331, 2006.

[18] H. Yan, J. Maser, A. Macrander et al., “Takagi-Taupin description of X-Ray dynamical diffraction from diffractive optics with large numerical aperture," Physical Review B, vol. 76, no. 11, Article ID 115438, 13 pages, 2007.

[19] E. Spiller, Soft X-Ray Optics, SPIE Optical Engineering Press, Bellingham, Wash, USA, 1994.

[20] H. Yan, H. C. Kang, J. Maser et al., "Characterization of a multilayer Laue lens with imperfections," Nuclear Instruments and Methods in Physics Research, Section A: Accelerators, Spectrometers, Detectors and Associated Equipment, vol. 582, no. 1, pp. 126-128, 2007.

[21] H. Yan, "X-Ray dynamical diffraction from multilayer Laue lenses with rough interfaces," Physical Review B, vol. 79, no. 16, Article ID 165410, 12 pages, 2009.

[22] R. Conley, C. Liu, C. M. Kewish, A. T. Macrander, and C. Morawe, "Multilayer growth in the APS rotary deposition system," in Advances in X-Ray/EUV Optics and Components II, vol. 6705 of Proceedings of SPIE, San Diego, Calif, USA, September 2007.

[23] http://www.msi-pse.com/Polaris.htm.

[24] C. Liu, R. Conley, and A. T. Macrander, "Film stress studies and the multilayer Laue lens project," in Advances in $X$ Ray/EUV Optics, Components, and Applications, vol. 6317 of Proceedings of SPIE, San Diego, Calif, USA, August 2006.

[25] Y. P. Wang, H. Zhou, L. Zhou, R.L. Headrick, A.T. Macrander, and A.S. Ozcan, "Interface roughness evolution in sputtered $\mathrm{WSi}_{2}$ /Si multilayers," Journal of Applied Physics, vol. 101, no. 2 , Article ID 023503, 6 pages, 2007.

[26] T. Koyama, T. Tsuji, H. Takano et al., "Performance test of $\mathrm{Mo} / \mathrm{Si}$ and $\mathrm{Mosi}_{2} / \mathrm{Si}$ multilayer Laue lenses," Journal of Physics: Conference Series, vol. 186, no. 1, Article ID 012066, 2009.

[27] T. Koyama, S. Ichimaru, T. Tsuji et al., "Optical properties of MoSi2/Si multilayer laue lens as nanometer X-Ray focusing device," Applied Physics Express, vol. 1, no. 11, Article ID 117003, 3 pages, 2008.

[28] R. Conley, C. Liu, J. Qian et al., "Wedged multilayer Laue lens," Review of Scientific Instruments, vol. 79, no. 5, Article ID 053104, 4 pages, 2008.

[29] N. Jahedi, R. Conley, B. Shi, J. Qian, K. Lauer, and A. Macrander, "Metrology of multilayer Laue lens structures by means of scanning electron microscope imaging," Nuclear Instruments and Methods in Physics Research, Section A: Accelerators, Spectrometers, Detectors and Associated Equipment, vol. 616, no. 2-3, pp. 89-92, 2010.

[30] H. C. Kang, G. B. Stephenson, C. Liu et al., "Sectioning of multilayers to make a multilayer Laue lens," Review of Scientific Instruments, vol. 78, no. 4, Article ID 046103, 3 pages, 2007.

[31] R. Conley, N. Boueta, J. Biancarosaa et al., "The NSLS-II multilayer laue lens deposition system," in Advances in $X$ Ray/EUV Optics and Components IV, vol. 7448 of Proceedings of SPIE, San Diego, Calif, USA, August 2009.

[32] A. Authier, Dynamical Theory of X-ray Diffraction, Oxford University Press, Oxford, UK, 2002.

[33] NSLS-II conceptual report, http://www.bnl.gov/nsls2/project/. 
[34] H. Yan, J. Maser, H. C. Kang, A. Macrander, and B. Stephenson, "A theoretical study of two-dimensional point focusing by two multilayer Laue lenses," in Advances in X-Ray/EUV Optics and Components III, vol. 7077 of Proceedings of SPIE, San Diego, Calif, USA, August 2008.

[35] D. Shu, H. Yan, and J. M. Maser, in Proceedings of SRI-2008 (to be published in Nucl. Instrum. Methods A).

[36] D. Shu, H. Yan, and J. M. Maser, USA Patent No. 7597475.

[37] H. Yan, V. Rose, D. Shu et al., submitted. 